\title{
Carcinoma urotelial de vejiga estadio T1: subestadiaje, patrones morfológicos de invasión y su significado pronóstico
}

\author{
Trias I*, Orsola $\mathrm{A}^{* * *}$, Español I*, Vidal N*, Raventós CX***, Bucar S**. \\ *Servicio de Anatomía Patológica. Clínica Plató Fundació Privada. Barcelona. ${ }^{* *}$ Servicio de Urología. \\ Clínica Plató Fundació Privada. Barcelona. ***Servicio de Urología Hospital Vall d'Hebrón. Barcelona.
}

Actas Urol Esp. 2007;31(9):1002-1008

\section{RESUMEN}

CARCINOMA UROTELIAL DE VEJIGA ESTADIO T1: SUBESTADIAJE, PATRONES MORFOLÓGICOS DE INVASIÓN Y SU SIGNIFICADO PRONÓSTICO

Desde 1990 en que se publicaron las primeras series sobre subestadiaje, han aparecido numerosas publicaciones sobre el subnivel de invasión de los carcinomas de alto grado T1. La invasión profunda conlleva un elevado riesgo de progresión (alrededor del 30-35\% de casos progresan) frente a los casos de invasión superficial por encima de la muscularis mucosae, en los que la progresión se encuentra alrededor del $10 \%$, por lo que para la mayoria de autores vale la pena tener en cuenta los subT1, en el manejo del paciente. En esta revisión se presentan las series más exhaustivas que han valorado el subestadiaje y se valoran los diferentes métodos de efectuar esta estadificación teniendo en cuenta la dificultad inherente a las muestras que proceden de resección transuretral (RTU).

Palabras clave: Carcinoma urotelial T1 Subestadiaje

\section{ABSTRACT}

BLADDER UROTHELIAL CARCINOMA STAGE T1: SUBSTAGING, INVASION MORPHOLOGICAL PATTERNS AND ITS PROGNOSIS SIGNIFICANCE

Since 1990 when the first series on substaging were published, they have published numerous publications on the invasion sublevel of high degree T1 carcinomas. The deep invasion entails a high risk of progression (around 30-35\% of cases progress) as opposed to the cases of superficial invasion over "muscularis mucosae", in which the progression is around $10 \%$, reason why most authors consider subT1, in patient management. In this revision the more exhaustive series that have evaluated substaging are shown and also the different methods to carry out this staging considering the inherent difficulty to the samples that come from transurethral resection (RTU).

Keywords: Urothelial Carcinoma T1 Substage.

A lrededor del 70\% de los nuevos casos de neoplasias vesicales, son carcinomas papilares uroteliales sin invasión (Ta), o que invaden el tejido subepitelial sin alcanzar la capa muscular y se caracterizan por su alta capacidad de recidiva ${ }^{1,2}$. Los estadios más iniciales (especialmente los Ta y T1) se consideran grupos de bajo riesgo y en el ambiente urológico se les denomina tumores superficiales, aunque este término esté cada vez más discutido ${ }^{3}$. Esta agrupación conceptual separa de manera drástica los casos de alto riesgo de muerte por enfermedad (T2 en adelante) de los que en principio se comportan de manera distinta, con tendencia a la recidiva y en teoría bajo riesgo de progresión. Sin embargo se están agrupando tumores con distintos grados (alto y bajo grado), distintos estadios (Tis, Ta y T1) y también distintos comportamientos ya que entre un $10 \mathrm{y}$ un $40 \%$ de estos tumores progresan en estadio ${ }^{4-7}$. Por lo tanto es lógico que uno de los objetivos 
más deseados de los urólogos sea encontrar aquellos datos clave que permitan diferenciar los casos de tumores no infiltrantes de la muscular propia (TNIMP) con elevado riesgo de progresión ${ }^{7}$. La literatura está llena de estudios destinados a identificar los llamados factores de riesgo de los carcinomas superficiales ${ }^{7,8}$. En esta revisión intentaremos analizar los más relevantes haciendo hincapié en los que se refieren al subestadiaje de los carcinomas T1 de vejiga.

\section{LOS CARCINOMAS SUPERFICIALES DE VEJIGA}

Esta definición abarca a los Tis, Ta y T1 para cualquier grado y son, en realidad, la mayoría de los casos de tumor vesical que cualquier urólogo deberá tratar durante su vida profesional. Como grupo, se caracterizan por tendencia a la recidiva (varía entre un 14 a un $70 \%)^{7,9}$ y una probabilidad de progresión a los 5 años que oscila entre el 7 y el $40 \%{ }^{7}$. Esta gran variación en el comportamiento refleja su heterogeneidad, y la dificultad y confusión a la hora de definir los grupos de riesgo. Además de mezclar grados y estadios se añade la falta de unidad de criterios en el momento de valorar la evolución de estos tumores lo cual hace poco comparables muchos de los trabajos publicados en la literatura . En realidad se trata de separar aquellos casos que en su historia natural solamente recidivarán sin progresar, de los que progresarán en estadio a lo largo de futuras recidivas. Así, los factores asociados a un mayor riesgo de recidiva local superficial no son necesariamente los mismos que los asociados a mayor riesgo de progresión, aunque algunos de los factores estén compartidos.

\section{Factores asociados a recidiva}

Varios trabajos han encontrado varias circunstancias relacionadas con las recidivas, o mejor, con el tiempo libre hasta la primera recidiva, así la multiplicidad de tumores, el tamaño del tumor superior a $3 \mathrm{~cm}$, el tratamiento con BCG y la ratio de recidivas anteriores/año son factores de riesgo reconocidos ${ }^{7,8}$.

Parece ser que ni el estadio, ni el grado ni el estatus del tumor estudiado (recurrente o primario) se relacionan con las recidivas ${ }^{5,7,8}$.

\section{Factores relacionados a progresión}

Aunque la mayoría de los trabajos detectan mejor el riesgo de recidiva que el de progresión ${ }^{8}$, hay algunos datos que parecen tener una clara relación con la progresión. Así vuelven a aparecer la multiplicidad de lesiones y el tamaño superior a $3 \mathrm{~cm}$ sin embargo adquieren más importancia el grado (grado 3), el estadio (categoría T1), la existencia de carcinoma in situ y la respuesta al tratamiento ${ }^{5,7}$. En general está aceptado que el subgrupo alto grado (G3 OMS 1973) ${ }^{10} \mathrm{~T} 1$ es uno de los que presenta mayor riesgo con un índice de progresión que oscila entre el 10 y el $54 \% \%^{8,11-}$ 13. Debemos recordar que cuando se habla de progresión en un carcinoma urotelial T1 se está indicando el hecho que se transforma en invasor de capa muscular (T2), con lo que el riesgo de muerte por enfermedad aumenta significativamente obligando a cambiar la actitud terapéutica. Es en este contexto donde los estudios del subestadiaje han encontrado su sitio como factor predictivo.

\section{EL SUBESTADIAJE}

El subestadiaje se basa en el reconocimiento de la existencia de una cierta estratificación de la mucosa vesical con lo que es necesario conocer bien la histología de la misma.

\section{Microanatomía de la vejiga urinaria}

La vejiga urinaria se divide básicamente en dos áreas: la muscular propia y la mucosa. La muscular propia se caracteriza por fascículos entrelazados de fibras musculares lisas. Un conjunto de fascículos constituyen un haz muscular y un conjunto de haces constituye una capa muscular. En la vejiga se reconocen fácilmente dos capas (externa e interna) aunque se describen tres especialmente en el cuello vesical ${ }^{14}$. Entre los haces musculares es posible observar tejido conectivo y adiposo cuya proporción aumenta con la edad lo cual conviene tener en cuenta a la hora de determinar el nivel de invasión en muestras de tumor vesical por RTU, y no interpretar la existencia de tejido adiposo necesariamente como representación de tejido extravesical ${ }^{15}$.

El término mucosa vesical se refiere al epitelio, a la lámina propia (espacio de tejido conectivo entre la membrana basal del epitelio y la 
muscularis mucosae), la muscularis mucosae y la submucosa (Fig. 1). El epitelio de la vejiga suele ser de la variedad transicional (urotelio) y se sustenta sobre una membrana basal que actúa de barrera funcional entre el tejido subepitelial y el urotelio. Dixon y Gosling ${ }^{16}$ publicaron en una revista de anatomía la existencia de una muscularis mucosae en la submucosa de la vejiga urinaria. Se describe como unos finos haces musculares que, de forma discontinua, se disponen paralelamente a la membrana basal y se acompañan de vasos cuyas paredes tienen cierto grosor. Finalmente la submucosa es el tejido conectivo que se sitúa entre la muscularis mucosae y la muscular propia (Fig. 1).

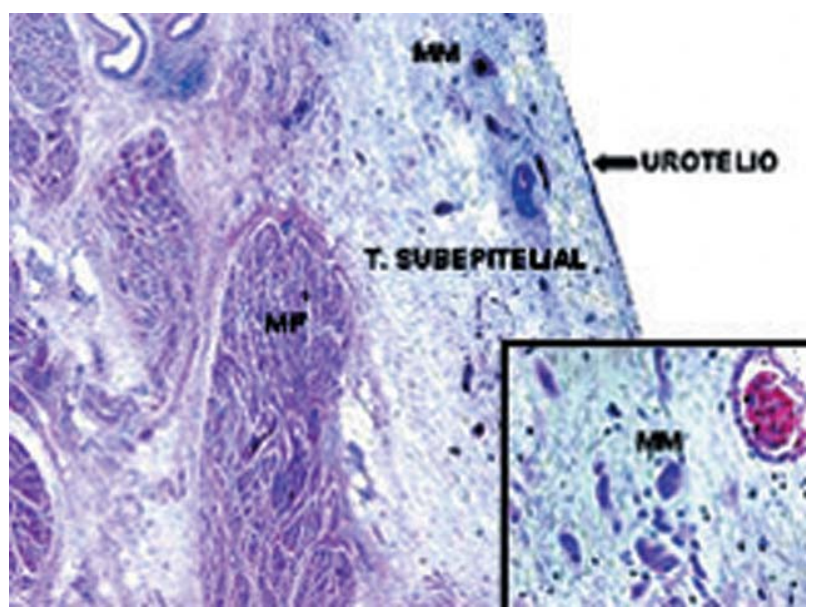

FIGURA 1. Microanatomía normal de mucosa y pared de la vejiga con urotelio (señalado por una flecha), el tejido subepitelial con finas fibras de musculares mucosae que se aprecian mejor a mayor aumento, y las fibras mucho más gruesas de la muscular propia. Hematoxilina Eosina x4 (imagen insertada x40). MM: Muscularis mucosae MP:Muscular propia T.Subepitelial: Tejido subepitelial.

\section{Variaciones de la normalidad de la muscularis mucosae}

Aunque ya se ha mencionado que la muscularis mucosae es discontinua, vale la pena insistir en ello y en otras características que los patólogos deben conocer dado el valor que tiene tanto en el subestadiaje, como se verá más adelante, como para no confundir muscular propia con muscularis mucosae y por lo tanto, interpretar como un T2 lo que en realidad es T1. En 1987 Ro et al. ${ }^{17}$ realizaron un magnífico estudio en el que demostraron la gran variabilidad de la muscularis mucosae. Así ellos demostraron que esta capa es continua sólo en el 3\% de los casos, es discontinua en la mayoría con finas fibras dispersas y que en el 6\% no existe. Además no la identificaron en la zona del trígono. Por otra parte, recientemente Paner et al. ${ }^{18}$ describen variaciones importantes, confirmando la dificultad en identificar la muscularis mucosae en la zona del trígono y diferenciarla de fibras de muscular propia ya que en esta zona, existen prolongaciones en forma de finas fibras musculares provenientes de la muscular propia, que se extienden de manera perpendicular a la superficie epitelial llegando a alcanzar una situación casi suburotelial (Fig. 2). En el resto de la vejiga, estos autores describen la muscularis mucosae como la existencia de finos haces musculares paralelos a la superficie, visibles en el 37$79 \%$ de los casos estudiados. En algunos casos estos "finos" haces musculares son hipertróficos y pueden llevar a confusión con la muscular propia. Se encuentran mayoritariamente en la cúpula (Fig. 3), pero la disposición ligeramente desordenada, la topografia de la fibra muscular situada de manera evidente en la lamina propia así como la comparación con inequívocos haces de capa muscular propia, deben ayudarnos a realizar una correcta interpretación y evitar un sobrediagnóstico en el caso de invasión por carcinoma.

\section{El subestadiaje}

En 1990 Younes et $\mathrm{al}^{19}$ iniciaron una subclasificación de los T1 basándose en la existencia de una mucosa que estaría dividida en tres partes:

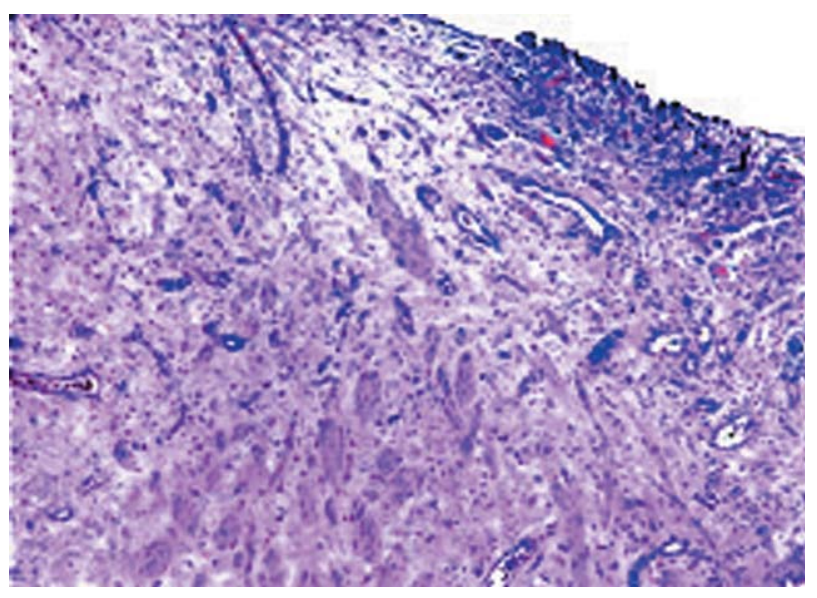

FIGURA 2. H\&E x10. Zona de trígono con finas fibras de muscular que se acercan a la mucosa sin que se identifique una muscularis mucosae. 


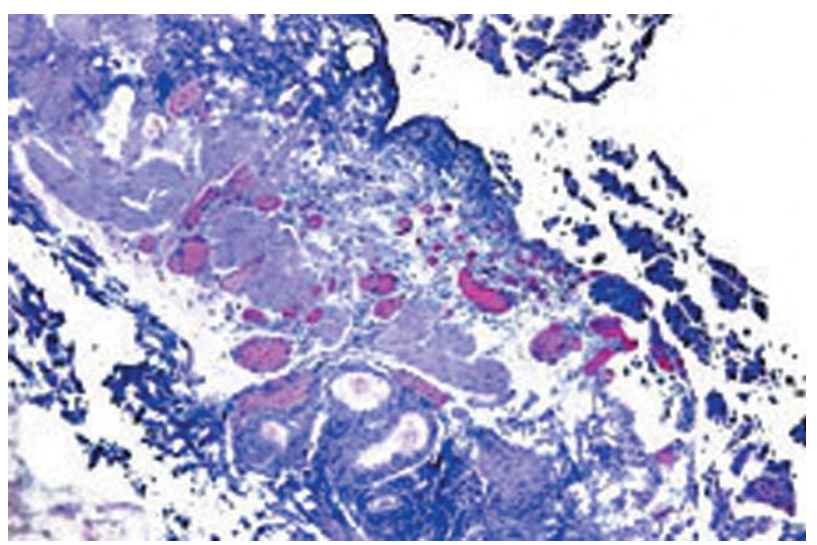

FIGURA 3. H \& E x10. Muscularis mucosae hipertrófica.

a) la zona situada entre la membrana basal y la muscularis mucosae, b) la zona de la muscularis mucosae y c) la zona entre la muscularis mucosae y la muscular propia (Fig. 4). A los tumores que invadían la primera zona los clasificaron como Tla, los de la segunda como T1b y la tercera y más profunda como T1c. Demostraron unas diferencias de sobrevida a los 5 años del 10\% para los Tlc y del 75\% para los Tla y Tlb. A partir de esta publicación ha habido múltiples revisiones que demuestran lo mismo con definiciones ligeramente distintas para cada subT1, dado que no hay un consenso para definir el subestadiaje. Estas publicaciones con sus criterios y resultados, se resumen en la Tabla $1^{4,6,19-27}$ y con alguna excepción, en todas se demuestra una relación estrecha entre invasión profunda (o sea desde la muscularis mucosae en adelante) y progresión. Frente al 20\% de progresión para todos los tumores grado 3 (OMS 1973) T1 considerados globalmente y $\sin$ subestadiar $^{5}$, la mayoría de las publicaciones encuentran diferencias entre los Tla (progresan alrededor de un 10\%) y los T1b y c (progresan entre un 29-53\%) (Tabla 1). Está claro que somos capaces de discriminar grupos de bajo riesgo (Tla) de los de alto riesgo (T1b y c) lo cual justifica modificar el manejo de estos pacientes con abordajes terapéuticos y de seguimiento diferenciados.

Teniendo en cuenta la gran variabilidad de la muscularis mucosae (ver apartado anterior) sorprende la gran cantidad de trabajos que siguen la clasificación inicial de Younes $^{19}$ con algunas variaciones, y las escasas publicaciones con micrómetros $^{9,24}$ (Tabla 1). Probablemente esto es
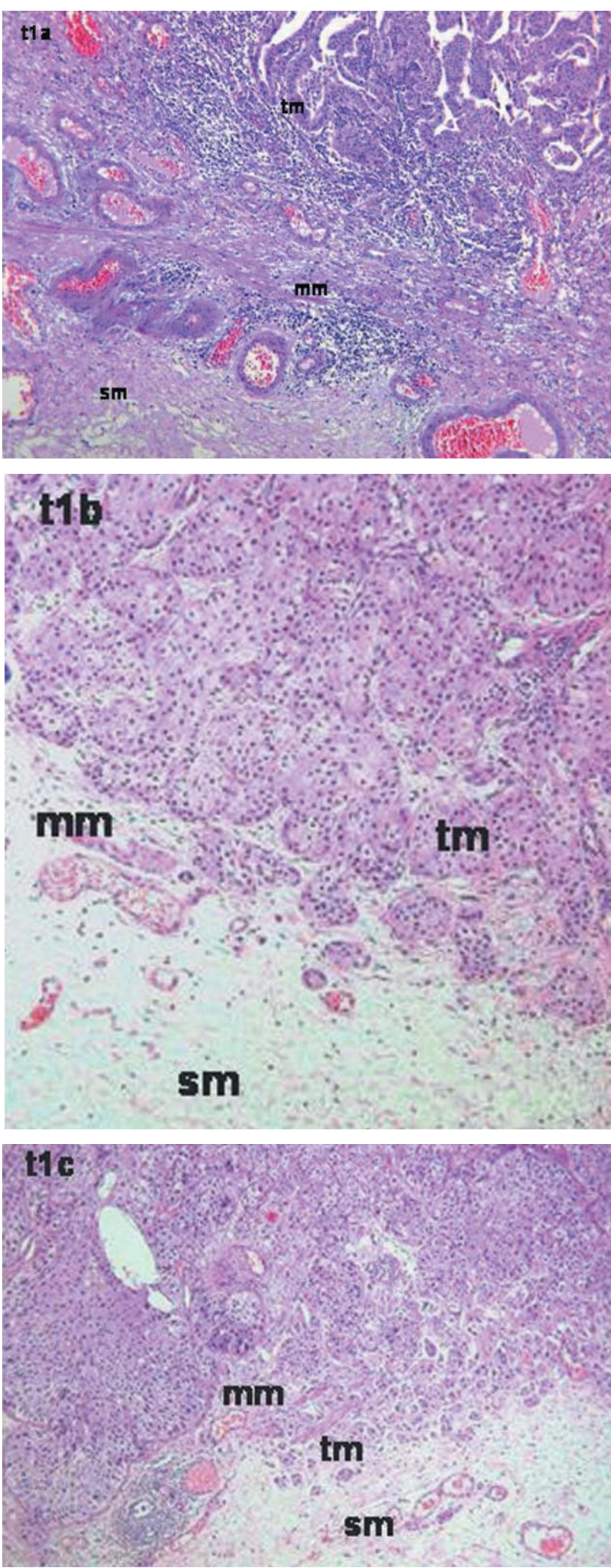

FIGURA 4 a. H\&E T1a. tm: tumor, mm: musculares muco sae, sm: submucosa. H\&E T1b. El tumor alcanza el nivel de la musuclaris mucosae. tm: tumor, mm: musculares mucosae, sm: submucosa. T1c H\&E x10. Se aprecia tumor por encima y por debajo de la muscularis mucosae. $\mathrm{mm}$ : musuclaris mucosa , tm: tumor, sm: submucosa. 
Tabla 1

\begin{tabular}{|c|c|c|c|c|c|c|}
\hline Año & Autor & Ref. & Estadiaje* & № Casos & Progresión & Sobrevida \\
\hline 1990 & Younes et al. & Cancer 66 & $\begin{array}{l}\text { Tla(lp) } \\
\text { T1b(mm) } \\
\text { T1c(sm) }\end{array}$ & $\begin{array}{c}15 \\
3 \\
14\end{array}$ & & $\begin{array}{l}75 \% \\
10 \%\end{array}$ \\
\hline 1994 & Hasuie et al. & Urology 43 & $\begin{array}{l}\mathrm{T} 1 \mathrm{a}(\mathrm{lp})^{* *} \\
\mathrm{~T} 1 \mathrm{~b}(\mathrm{~mm}+\mathrm{sm})\end{array}$ & $\begin{array}{l}60 \\
28\end{array}$ & $\begin{array}{c}6,7 \% \\
53,5 \%\end{array}$ & $\begin{array}{l}95 \% \\
82 \%\end{array}$ \\
\hline 1995 & Angulo et al. & Urology 45 & $\begin{array}{l}\text { Tla(lp+mm) } \\
\text { T1b(sm) }\end{array}$ & $\begin{array}{l}50 \\
49\end{array}$ & & $\begin{array}{l}86 \% \\
52 \%\end{array}$ \\
\hline 1997 & Holmäng et al. & J Urol. 147 & $\begin{array}{l}\text { Tla(lp) } \\
\text { T1b(mm+sm) }\end{array}$ & $\begin{array}{l}26 \\
38\end{array}$ & $\begin{array}{l}36 \% \\
58 \%\end{array}$ & $\begin{array}{l}58 \% \\
42 \%\end{array}$ \\
\hline 1998 & Smits et al. & Urology 52 & $\begin{array}{l}\text { Tla(lp) } \\
\text { T1b(mm) } \\
\text { Tlc(sm) }\end{array}$ & $\begin{array}{c}\text { Total: } 119 \\
119\end{array}$ & $\begin{array}{c}6 \% \\
33 \% \\
55 \%\end{array}$ & \\
\hline 1998 & Hermann et al. & J Urol. 148 & $\begin{array}{l}\text { Tla*** } \\
\text { T1b } \\
\text { Tlc }\end{array}$ & $\begin{array}{l}31 \\
60 \\
52\end{array}$ & $57 \%$ & $\begin{array}{l}79 \% \\
70 \%\end{array}$ \\
\hline 1999 & Cheng et al. & J Clin Oncol 16 & $\begin{array}{l}\text { T1(lp) } \\
\text { T1(mm+sm) }\end{array}$ & $\begin{array}{l}23 \\
21\end{array}$ & $\begin{array}{l}11 \% \\
32 \%\end{array}$ & \\
\hline 2000 & Kondylis et al. & J Urol 153 & $\begin{array}{l}\text { Tla(mm) } \\
\text { T1b(sm) }\end{array}$ & $\begin{array}{l}32 \\
17\end{array}$ & $\begin{array}{l}22 \% \\
29 \%\end{array}$ & \\
\hline 2001 & Bernardini et al. & J Urol. 155 & $\begin{array}{l}\text { Tla(lp) } \\
\text { Tlb(mm+sm) }\end{array}$ & $\begin{array}{l}54 \\
40\end{array}$ & $=$ & \\
\hline 2003 & Trias et al. & Rev Esp Patol. 36 & $\begin{array}{l}\text { Tla(lp) } \\
\text { Tlb(mm+sm) }\end{array}$ & $\begin{array}{l}11 \\
13\end{array}$ & $\begin{array}{c}9 \% \\
30,7 \%\end{array}$ & \\
\hline 2005 & Van der Aa et al. & Human Pathol. 53 & $\begin{array}{l}\text { T1(foc) } \\
\text { T1 (ext) }\end{array}$ & $\begin{array}{l}24 \\
29\end{array}$ & $\begin{array}{l}37,5 \% \\
65,5 \%\end{array}$ & \\
\hline 2005 & Orsola et al. & Eur Urol. 87 & $\begin{array}{l}\text { T1a(lp) } \\
\text { T1b(mm) } \\
\text { T1c(sm) }\end{array}$ & $\begin{array}{l}38 \\
10 \\
37\end{array}$ & $\begin{array}{c}8 \% \\
30 \% \\
35 \%\end{array}$ & \\
\hline
\end{tabular}

*Tipos de subestadiajes realizados, lp: Invasión de lámina propia, mm: Invasión hasta nivel de mm, sm: Invasión de submucosa.

**T1a: Invasión del eje fibrovascular de la papila, T1b: Invasión de lp por debajo del eje fibrovascular.

***T1a Invasión del eje fibrovascular por encima de la mm, T1b: Invasión por debajo del eje y por encima de la mm, T1c: Invasión por debajo de la mm. = Riesgo de progresión aumentado en un 7,5 si Carcinoma in situ asociado.

debido a la. naturaleza de las muestras que se manejan en las RTU que complican cualquier tipo de medición, pero especialmente las que se realizan con un micrómetro, siendo mucho más fácil utilizar la identificación de estructuras propias de la microanatomía (muscularis mucosae o el plexo vascular que la acompaña) que pueden orientar al patólogo sobre la zona de la submucosa que analiza. Nuestra experiencia empezó en el 1996 cuando de manera sistemática los patólogos del servicio intentamos subestadiar todos los
$\mathrm{T}^{4}$. Este análisis sistemático nos ayudó a introducir la disciplina del reconocimiento de la profundidad del tejido subepitelial a través de la muscularis mucosae o del plexo vascular. Este ejercicio nos permitió pasar de un $60 \%$ de casos en los que se pudo realizar el subestadiaje al inicio (1996-2001), hasta el $87,6 \%$ posteriormente $^{27}$. Estas cifras son parecidas a las que refleja la literatura ya que en los primeros trabajos se comunica un 65-72\% de casos en los que es posible efectuar el subestadiaje ${ }^{19-21}$ llegando hasta el 
80 o incluso el $90 \%$ en los más recientes ${ }^{6,23,25-26}$ Nuestra recomendación para el patólogo que se inicie en el subestadiaje, es que utilice los criterios de Younes ${ }^{19}$ por dos motivos: 1) son los más utilizados y por lo tanto los resultados se podrán comparar con otros y 2) obliga a la búsqueda sistemática de la muscularis mucosae o del plexo vascular y se gana en rigor diagnóstico.

\section{Críticas al subestadiaje}

1. La principal crítica es que no se puede realizar siempre y en la reproducibilidad que puede ser muy variable ${ }^{28,29}$. Esto es debido a la propia naturaleza de las muestras, al hecho de que hay zonas sin muscularis mucosae (trígono, Fig. 2) en que quizá no es posible clasificar el subT1 según Younes ${ }^{19} \mathrm{y}$ al artefacto que se observa frecuentemente en los fragmentos que se reciben. Este aspecto puede solucionarse parcialmente si el urólogo es consciente de las alteraciones que se producen en el tejido en el momento de obtener las muestras. También debe recordarse que el mejor subestadiaje es el que se hace en el primer episodio sin el artefacto de RTUs previas. Por otro lado si se consigue una definición consensuada, muchos de los problemas de reproducibilidad desaparecerán.

2. El infraestadiaje. La elevada cifra de casos infraestadiados en piezas de cistectomías preco$\mathrm{ces}^{30}$ plantea la posibilidad de que algunos casos de invasión profunda de submucosa sean en realidad invasiones de muscular no demostradas en el material de RTU. La respuesta a eso pasa por apelar al rigor tanto del urólogo que debe esforzarse en mandar muestras lo más preservadas posibles al anatomopatólogo, como del patólogo que no debe tener miedo a diagnosticar TX cuando en las muestras recibidas y siempre que se haya efectuado una inclusión total, no identifica muscular propia.

\section{CONCLUSIONES}

El subestadiaje es una información del riesgo, no una información de categoría definitiva, pero añade información que contribuye positivamente al manejo del enfermo.

Nuestra experiencia demuestra que es posible realizar este análisis desde un servicio de anatomía patológica general, simplemente hay que invertir cierto tiempo en el aprendizaje, pero tiene la gran ventaja de no necesitar ninguna técnica especial por lo que está al alcance de cualquier patólogo.

Frente a las ventajas del subestadiaje hay que aceptar que no es posible efectuarlo en el 100\% de los casos. El \% de casos en que esto es posible oscila mucho y probablemente depende de muchos factores por lo que es recomendable que cada centro valide sus resultados.

Finalmente vale la pena recordar que sólo es posible hacer un buen subestadiaje cuando las muestras incluyen muscular propia, se ha hecho una inclusión total del tejido extraído por el urólogo y los artefactos de resección son mínimos.

\section{REFERENCIAS}

1. UICC International Union Against Cancer. Sobin LH, Wittekind Ch Editors. TNM Classification of malignant tumours. 6th ed. New York: Wiley-Liss; 2002.

2. Amling CL, Thrasher HA, Dodge RK, Robertson JE, Paulson DF. Radical cystectomy for stages Ta, Tis and T1 transitional cell carcinoma of the bladder. J Urol. 1994; 151(1):31-35.

3. Soloway MS. Expectant treatment of small, recurrent, low-grade, non-invasive tumors of the urinary bladder. Urol Oncol. 2006;24(1):58-61.

4. Trias I, Español I, Raventos C, Orsola A, Paga J, Orsola I. Evolución del carcinoma papilar urotelial G3T1 según el nivel de invasión de la submucosa. Rev Esp Patol. 2003;4: 419-424.

5. Vicente J, Chechile G, Salvador J, editores. En tumores vesicales superficiales. Barcelona, Acción Médica SA 2000.

6. Smits G, Schaafsma E, Kiemeney L, Caris C, Debruyne F, Witjes JA. Microstaging of pT1 transitional cell carcinoma of the bladder: Identification of subgroups with distinct groups of progression. Urology. 1998;52(6):1009-1013.

7. Sylvester RJ, van der Meijden AP, Oosterlink W, Witjes JA, Bouffioux C, Denis L, et al. Predicting recurrence and progressions in individual patients with stage Ta T1 bladder cancer uring EORTC Risk Tables: A Combined Analysis of 2596 Patients from seven EORTC trials. Eur Urol. 2006; 49(3):466-475.

8. Millan-Rodriguez F, Chechile-Toniolo G, Salvador-Bayarri, Palou J, Algaba F, Vicente-Rodriguez J. Primary superficial bladder cancer risk groups according to progression, mortality and recurrence. J Urol. 164;2000(3Pt1):680-684.

9. Van der Aa MNM, van Leenders GJLH, Steyeberg AW, van Rhijn BW, Jöbsis AC, Zwarthoff AC et al. A new substaging pT1 papillary bladder cancer: a prognostic evaluation. Human Pathol. 2005;36(9):981-986.

10. Mostofi FK, Sobin LH, Torloni H. Histologic typing of urinary bladder tumors. En Mostofi FK, Sobin LH, Torloni H, editores en International Classification of Tumors. Ginebra Suiza 1973.

11. Pansadoro V, Emiliozzi P, Defidio L, Donadio D, Florio A, Maurelli S, et al. Bacillus Calmette-Guerin in the treatment of stage T1 grade 3 transitional cell carcinoma of the bladder: long term results. J Urol. 1995;154(6):2054-2058. 
12. Herr HW. Progression of stage T1 bladder tumors after intravesical bacillus Calmette-Guerin. J Urol. 1991;145(1): 40-43.

13. Pham HT, Soloway MS, High risk superficial bladder cancer: intravesical therapy for T1 G3 transitional carcinoma of the urinary bladder. Seminar Urol Oncol. 1997;15(3): 147-153.

14. Tumores vesicales. Morfobiología de las neoplasias superficiales de la vejiga urinaria, Ed Vicente, Chechile y Salvador pg 17. Barcelona: Acción Médica 2000.

15. Algaba F, Moreno A, Trias I, editores. En Uropatología no tumoral. Correlación morfológica, molecular y clinica. Capítulo II pg 92. Barcelona: Pulso ediciones S.A. 1997.

16. Dixon JS, Gosling JA. Histology and fine structure of the muscularis mucosae in the staging of invasive transitional carcinoma of the urinary bladder. J Anat. 1983;136(Pt2): 265-271.

17. Ro JY, Ayala AG, El-Nagger A. Muscularis mucosae of urinary bladder. Am J Surg Pathol. 1987;11(9):668-673.

18. Paner GP, Wojcik EM, Datta MW. Further characterization of the muscle layers of the urinary bladder by systematic histologic mapping: Implications for pathologic staging. Modern Pathology Annual Meeting Abstracts 2006;19:154.

19. Younes M, Sussman J, True LD. The usefulness of the level of the muscularis mucosae in the staging of invasive transitional cell carcinoma of the bladder. Cancer 1990; 66(3):543-548.

20. Hasui Y, OSada Y, Kitada S, Nishi S. Significance of invasion to the muscularis mucosae on the progression of superficial bladder cancer. Urology. 1994;43(6):782-786.

21. Angulo JC, Lopez JI, Grignon DJ, Sanchez-Chapado M. Muscularis mucosa differentiates two populations with different prognosis in stage T1 bladder cancer. Urology 1995; 45(1):47-53.

22. Holmäng S, Hedelin H, Anderström C, Holmberg E, Johansson SL. The importance of the depth of invasion in stage T1 bladder carcinoma: a prospective cohort study. J Urol. 1997;157(3):800-803.
23. Hermann CG, Horn T, Steven K. The influence of the level of lamina propia invasion and the prevalence of p53 nuclear accumulation on survival in stage $\mathrm{T} 1$ transitional cell bladder cancer. J Urol. 1998;159:91-94.

24. Cheng L, Neumann RM, Weaver AL, Spotts BE, Bostwick DG. Predicting cancer progression in patients with stage T1 bladder carcinoma. J Clin Oncol. 1999;17(10):31823187.

25. Kondylis FI, Demirci S, Ladaga L, Kolm, P, Schellhammer PF. Outcomes after intravesical bacillus Calmette-Guerin are not affected by substaging of high grade $\mathrm{T} 1$ transitional cell carcinoma. J Urol. 2000;163(4):1120-1123.

26. Bernardini S, Billerey C, Martin M, Adessi GL, Wallerand $\mathrm{H}$, Bittard $\mathrm{H}$. The predictive value of muscularis mucosae invasion and p53 over expression of stage T1 bladder carcinoma. J Urol. 2001;165(1):42-46.

27. Orsola A, Trias I, Raventos CX, Español I, Cecchini L, Bucar S, et al. Initial high-grade $\mathrm{T} 1$ urothelial cell carcinoma: feasibility and prognostic significance of lamina propria invasion microstaging $(\mathrm{T} 1 \mathrm{a} / \mathrm{b} / \mathrm{c})$ in $\mathrm{BCG}$-treated and BCG-non-treated patients. Eur Urol. 2005;48(2):231-238.

28. Platz CE, Cohen MB, Jones MP, Olson DB, Lynch CF. Is microstaging of early invasive cancer of the bladder possible and useful? Mod Pathol. 1996;9(11):1035-1039.

29. Murphy WM. Editorial comment on: The importance of the depth of invasion in stage T1 bladder carcinoma: a prospective cohort study. J Urol. 1997;157:804.

30. Chang SS, Cookson MS. Radical cystectomy for bladder cancer: the case for early intervention. Urol Clin N Am. 2005;32(2): 147-155.

Correspondencia autor: Dra. I. Trias

Servicio de Anatomía Patológica. Clínica Plató

Plató 21 - 08006 Barcelona

Tel.: 933069900

E-mail autor: anatomia.patologica@clinicaplato.com

Información artículo: Original - Vejiga 